\title{
PERMEADO DE SUERO COMO ABONO: RESPUESTA DE CULTIVOS Y CAMBIOS EN UN NATRUSTOL ${ }^{1}$
}

\author{
Badino, O. N. ${ }^{2}$; Felli, O. ${ }^{3}$ P Pllatti, M. A. ${ }^{3} ;$ \\ GhiBerto, P. J. ${ }^{3}$ \& MIRETTI, M. C. ${ }^{3}$
}

\begin{abstract}
RESUMEN
El permeado de suero (PS) podría usarse como abono y reponer nutrientes al suelo; pero su tenor salino y de sodio puede dañarlo. Se evaluó la respuesta de maíz para silo y alfalfa en Natrustol típico a dosis entre 30 y $120 \mathrm{~m}^{3} \mathrm{PS} / \mathrm{ha}$ en el centro este de Córdoba (Argentina). Se midió producción, propiedades físicas y químicas del suelo hasta $30 \mathrm{~cm}$. No se observó un incremento en la producción; sí en el contenido de Nt, P, Ca y Na intercambiable; sin diferencias en MO, CIC, K intercambiable, salinidad y $\mathrm{pH}$. El Nt y P son móviles en el perfil por lo que existe riesgo de contaminar la napa freática. Con las mayores dosis se observó que se deteriora la estabilidad de agregados, la conductividad hidráulica (Ks) y el intervalo hídrico óptimo (IHO). Se aconseja usar dosis de 30 a $60 \mathrm{~m}^{3} /$ ha de PS, midiendo la composición del mismo que es muy variable y monitorear Nt, P y Na en el perfil; así como Ks y la densidad para estimar el IHO.
\end{abstract}

Key words: maiz, alfalfa, intervalo hídrico óptimo.

\begin{abstract}
Whey Permeate as fertilizer: Response of crops and Natrustol soil changes.

Whey Permeate (PS) could be used as fertilizer and nutrients to the soil. Saline and sodium content can damage it. The response of silo corn and alfalfa was evaluated in Natrustol soil en the east center of Córdoba (Argentina). The doses was between 30 and $120 \mathrm{~m}^{3} \mathrm{PS} /$ ha. Production, physical and chemical soil properties were measured up to $30 \mathrm{~cm}$. Production not increase; the $\mathrm{Nt}, \mathrm{P}, \mathrm{Ca}$ and interchangeable $\mathrm{Na}$ increased. There were no differences in organic matter, cation
\end{abstract}

1.- Financiado por CA+ID 2011 (UNL) 20/C409 y PICT-2012-2691.

2.- Departamento de Producción Animal. Facultad de Ciencias Agrarias (UNL). Kreder 2805. (3080HOF)

Esperanza, provincia de Santa Fe. Telefax +54 (3496) 426400. Email: mpilatti@fca.unl.edu.ar

3.- Departamento Ciencias del Ambiente. Facultad de Ciencias Agrarias (UNL).

Manuscrito recibido el 12 de diciembre de 2018 y aceptado para su publicación el 4 de junio de 2019.

Badino, O.N.; Felli, O.; Pilatti, M.A.; Ghiberto, P.J. \& Miretti, M.C. Permeado de suero como abono: Respuesta de cultivos y cambios en un natrustol. FAVE - Ciencias Agrarias 18 (1): 7-24.

CC BY-NC-SA 4.0 (c) (i) (2) 
exchange capacity, interchangeable $\mathrm{K}$; salinity and $\mathrm{pH}$. The $\mathrm{Nt}$ and $\mathrm{P}$ are mobile in the soil profile: risk of groundwater contamination. The highest doses deteriorate the stability of aggregates, the hydraulic conductivity (Ks) and the optimal water interval (IHO). It is recommended doses from 30 to $60 \mathrm{~m}^{3}$ / ha of PS, measuring PS composition, monitor Nt, P, Na in the soil profile, Ks and the density to estimate the IHO.

Key words: corn, alfalfa, optimal water interval.

\section{INTRODUCCIÓN}

La valorización agrícola de subproductos, permite un uso eficiente y reduce el impacto ambiental negativo que genera su disposición no controlada. El permeado de suero (PS) es un subproducto de la industria láctea, se obtiene del proceso de concentración de proteínas del suero (26), es rico en lactosa y sales minerales $(9,26)$. Contiene $\mathrm{N}, \mathrm{P}, \mathrm{K}, \mathrm{Ca}, \mathrm{Mg}$ y S , siendo utilizado como abono de suelos en Estados Unidos de Norte América, Canadá y Nueva Zelanda (10), en Argentina se desconoce ese uso excepto por el trabajo de Badino et al. (3).

El PS contiene también $\mathrm{Na}, \mathrm{Cl}$, sales, olores y acidez no deseables, limitando su utilización como abono, por esto existen restricciones para su aplicación e indicaciones para su monitoreo. Para tal fin Estados Unidos cuenta con la Agencia de Protección Ambiental (4) y Canadá tiene una Guía de Valorización de las Materias Residuales que se utilizan para fertilización de suelos (10). En Argentina, no existe normativa específica para el tema. No obstante se deben contemplar los requisitos establecidos a nivel provincial como en Santa Fe (Ley 11.717 de Medio Ambiente y Desarrollo Sustentable) y Córdoba (Ley 7.343, de Impacto Ambiental).
Las hipótesis del presente trabajo fue que la aplicación superficial de PS incrementa la producción de los cultivos y permite reponer nutrientes al suelo, tomando en consideración el $\mathrm{Na}, \mathrm{Cl}$, sales que pueden tener un efecto negativo sobre las propiedades del suelo.

El objetivo de la investigación fue evaluar la respuesta productiva a la adición de dosis crecientes de PS de la secuencia maíz para silo (Zea maíz) -alfalfa (Medicago sativa) en un Natrustol típico del centro este de Córdoba (Argentina) y medir los cambios químicos y físicos en el horizonte superficial.

\section{MATERIALES Y MÉTODOS}

El ensayo se realizó en el distrito de Arroyo Algodón Lat. sur $32^{\circ} 12^{\prime} 20^{\prime \prime}$ y Long. oeste $63^{\circ} 03^{\prime} 53^{\prime \prime}$, en el centro este de Córdoba (Argentina), en un Natrustol típico serie La Playosa (12), durante dos años consecutivos. Se sembró maíz para silo híbrido AX 890 CL (Nidera), con densidad de 80.000 semillas/ha; luego $10 \mathrm{~kg} / \mathrm{ha}$ de alfalfa Meca G9 (Asgrow). El PS se asperjó 7 días antes de la siembra de cada cultivo realizándose los análisis que se detallan en el Tabla 1. 
Respuesta de cultivos y suelo al permeado de suero

Tabla 1: Determinaciones analiticas realizadas al permeado de suero para su caracterización según APHA (2).

\begin{tabular}{|l|l|}
\hline Determinaciones analíticas & \multicolumn{1}{|c|}{ Técnica } \\
\hline $\mathrm{pH}$ & Método electrométrico. Potenciométrico \\
\hline Conductividad $(\mathrm{mS} / \mathrm{cm})\left(20^{\circ} \mathrm{C}\right)$ & Conductímetro \\
\hline Sólidos Totales $(\mathrm{g} / \mathrm{L})$ & Secado en estufa $105^{\circ} \mathrm{C}$ \\
\hline Nitrógeno Total $(\mathrm{mg} \mathrm{N} / \mathrm{L})$ & Método de Kjeldhal \\
\hline Fósforo Total $(\mathrm{mg} \mathrm{P} / \mathrm{L})$ & Colorimetría de ácido ascórbico \\
\hline Cloruros $(\mathrm{mg} / \mathrm{L} \mathrm{Cl})$ & Titulación argentométrica \\
\hline Sulfatos $\left(\mathrm{mg} / \mathrm{L} \mathrm{SO}{ }^{-2}\right)$ & Turbidimetría \\
\hline Sodio (mg/L Na) & Fotometría de llama \\
\hline Potasio $(\mathrm{mg} / \mathrm{L} \mathrm{K})$ & Fotometría de llama \\
\hline Calcio (mg/L Ca) & Espectrometría de absorción atómica de (FAAS) \\
\hline Magnesio (mg/L Mg) & Espectrometría de absorción atómica de (FAAS) \\
\hline
\end{tabular}

\section{Tratamientos:}

A continuación se detalla el tipo de tratamientos con el que se trabajó en el presente estudio.

Maíz para silo: Fertilización de base a todo el ensayo con $60 \mathrm{~kg} \mathrm{~N} / \mathrm{ha}$ (Sol Uan), instalándose 5 tratamientos en parcelas de $50 \mathrm{~m}$ por $30 \mathrm{~m}$ con 6 repeticiones (bloques totalmente aleatorizados). T: Testigo sin aplicación de PS; PS30: $30 \mathrm{~m}^{3} \mathrm{PS} / \mathrm{ha}$; P60: 60 $\mathrm{m}^{3} \mathrm{PS} / \mathrm{ha}$; PS120: $120 \mathrm{~m}^{3} \mathrm{PS} /$ ha y FDA: $100 \mathrm{~kg}$ de fosfato diamónico/ha, dosis de uso frecuente en la zona.

Alfalfa: Luego del maíz en el mismo sitio, con iguales tratamientos y repeticiones, sin fertilización de base. La dosis mayor de PS se redujo a PS90: $90 \mathrm{~m}^{3} \mathrm{PS} / \mathrm{ha}$

En ambos cultivos se realizó siembra directa.

\section{Mediciones en cultivos:}

En maíz, se determinó el rendimiento de biomasa aérea (MS/ha) y grano (kg grano/ ha). Se cosecharon manualmente 6 submuestras de $3 \mathrm{~m}^{2}$ en cada bloque. En alfalfa, se determinó la producción de biomasa aérea (MS/ha), cortes de $0,25 \mathrm{~m}^{2}$ con 8 submuestras por bloque, antes de cada pastoreo, totalizándose 7 cortes en 17 meses a partir de la siembra.

\section{Mediciones en suelo:}

Químicas: Se tomaron muestras compuestas por 30 submuestras a distintas profundidades $0-5 ; 5-15 ; 15-30 \mathrm{~cm}$, (a) 30 días antes de la aplicación del PS, (b) después de la cosecha del cultivo anual y (c) a los 17 meses de implantada la alfalfa. Las técnicas utilizadas en el análisis de suelo se detallan en la Tabla 2. 


\section{O. Badino et al.}

Tabla 2: Técnicas analíticas de suelos, ensayo de permeado de suero en maíz para silo y alfalfa en un Natrustol típico del centro este de Córdoba - Argentina.

\begin{tabular}{|c|c|c|c|}
\hline Determinaciones & Símbolo & Método & Fuente bibliográfica \\
\hline Materia orgánica total & $\mathrm{MO}$ & $\begin{array}{c}\text { Combustión húmeda (C x 1,724) } \\
\text { (Factor de recuperación } 0,77)\end{array}$ & (13) \\
\hline Nitrógeno total & $\mathrm{Nt}$ & Kjeldahl & (13) \\
\hline Reacción del suelo & $\mathrm{pH}$ & Potenciometría, relación 1:2,5 & (13) \\
\hline Fósforo extraíble & $\mathrm{P}$ & Bray Kurtz nro. 1 & (13) \\
\hline $\begin{array}{l}\text { Sodio y Potasio } \\
\text { intercambiable }\end{array}$ & Nai Ki & Extrac. acetato de amonio $1 \mathrm{~N}, \mathrm{pH} 7$ & (25) \\
\hline $\begin{array}{l}\text { Calcio y Magnesio } \\
\text { Intercambiable }\end{array}$ & Cai Mgi & Complexometría & (25) \\
\hline $\begin{array}{l}\text { Capacidad de } \\
\text { Intercambio catiónico }\end{array}$ & $\mathrm{CIC}$ & $\begin{array}{l}\text { Por desplazamiento del } \mathrm{NH}_{4}{ }^{+} \text {con } \\
\mathrm{Cl}_{2} \mathrm{Ca} \text { y éste con } \mathrm{CINa} \text {. }\end{array}$ & (25) \\
\hline $\begin{array}{l}\text { Conductividad eléctrica } \\
\text { extracto de saturación }\end{array}$ & CEes & Conductimetría & (24) \\
\hline
\end{tabular}

Tabla 3: Coeficientes de variación (\%) de propiedades edáficas según distintas fuentes de información.

\begin{tabular}{|c|c|c|c|c|c|c|c|c|c|c|}
\hline $\begin{array}{c}\text { Propiedad } \\
\text { Fuente }\end{array}$ & $\mathrm{pH}$ & $\mathrm{P}$ & $\mathrm{MO}$ & $\mathrm{Nt}$ & $\mathrm{ClC}$ & $\mathrm{Cai}$ & $\mathrm{Mgi}$ & $\mathrm{Ki}$ & Nai & CEes \\
\hline Internacional $\left({ }^{*}\right)$ & $\begin{array}{c}2 \mathrm{a} \\
15\end{array}$ & $\begin{array}{c}39 \mathrm{a} \\
157\end{array}$ & 16 a 41 & & $\begin{array}{c}19 \mathrm{a} \\
32\end{array}$ & $\begin{array}{c}31 \mathrm{a} \\
44\end{array}$ & & $\begin{array}{c}51 \mathrm{a} \\
53\end{array}$ & & 91 a 263 \\
\hline Local $\left({ }^{*}\right)$ & $4 \mathrm{a} 8$ & $7 \mathrm{a} 65$ & $3 \mathrm{a} 30$ & $\begin{array}{c}15 \mathrm{a} \\
25\end{array}$ & $\begin{array}{c}3 \mathrm{a} 21 \\
2 \mathrm{a} 24\end{array}$ & $\begin{array}{c}27 \mathrm{a} \\
40\end{array}$ & $\begin{array}{c}4 \mathrm{a} 43 \\
13 \mathrm{a}\end{array}$ & $\begin{array}{c}108 \mathrm{a} \\
74\end{array}$ \\
\hline $\begin{array}{c}\text { Adoptado en este } \\
\text { trabajo }\end{array}$ & 8 & 65 & 30 & 25 & 21 & 24 & 40 & 43 & 74 & 142 \\
\hline
\end{tabular}

(*) (11); (17) (**) Todos son Molisoles, algunos muestreos a nivel de grilla en lotes, otros varios lotes a nivel regional.

Criterio de comparación: Para comparar el estado del horizonte superficial se dispone sólo del valor de una muestra compuesta por 30 submuestras. Se usa la distribución " $t$ " para una probabilidad de 95\%, asumiéndose valores típicos del coeficiente de variación $(\mathrm{CV})$ para cada propiedad (Tabla 3). Se consideran significativas las diferencias entre los valores de cada muestra compuesta respecto del error típico cuando la diferencia de medias es superior al valor tabulado para la "t" (15).
Físicas: Las determinaciones físicas de suelo se presentan en el Tabla 4. Las muestras fueron extraídas de los mismos sitios y en los momentos establecidos para las determinaciones químicas. La toma de muestras de suelos se efectuó de 0 a $5 \mathrm{~cm}$; la estabilidad de agregados se evaluó en 3 muestras superficiales, inalteradas, compuestas por 20 submuestras cada una. Las demás mediciones se hicieron en muestras inalteradas: 15 cilindros, $5 \mathrm{~cm}$ altura y 100 $\mathrm{cm}^{3}$ de capacidad. 
Respuesta de cultivos y suelo al permeado de suero

Tabla 4: Determinaciones fisicas y técnicas de suelos utilizadas en ensayo de permeado de suero en maíz para silo y alfalfa en un Natrustol típico del centro este de Córdoba, Argentina.

\begin{tabular}{|c|c|c|c|}
\hline Determinaciones & Símbolo & Método & $\begin{array}{c}\text { Fuente } \\
\text { bibliográfica }\end{array}$ \\
\hline $\begin{array}{l}\text { Estabilidad de } \\
\text { agregados }\end{array}$ & $\begin{array}{l}\text { Ea } \\
\text { Eol }\end{array}$ & $\begin{array}{l}\text { Tamizado en agua, por triplicado } \\
\text { Pretratamiento con alcohol }\end{array}$ & $(16)$ \\
\hline Agua saturación & $\theta_{\mathrm{s}}$ & Por cálculo : $\theta_{s}=1-(\delta \mathrm{s} / \delta \mathrm{p})$ & \\
\hline $\begin{array}{l}\text { Agua cap.campo, } 10 \\
\mathrm{kPa}\end{array}$ & $\theta_{\mathrm{cc}}$ & Cámara de presión Richards & (14) \\
\hline $\begin{array}{l}\text { Cont. hídrico aireación } \\
\text { no es limitante }\end{array}$ & $\theta_{\mathrm{a}}$ & Por cálculo $\theta_{a}=\theta_{s}-0,15$ & (22) \\
\hline Densidad del suelo & $\delta s$ & Cilindro $100 \mathrm{~cm}^{3}, 15$ repetic. & (6) \\
\hline Densidad de partícula & $\delta p$ & Por cálculo en f(MO) & (20) \\
\hline $\begin{array}{l}\text { Límite agua fácilm. } \\
\text { disponible }\end{array}$ & $\begin{array}{c}\theta_{\mathrm{Fu}} \\
1700 \mathrm{kPa}\end{array}$ & $\begin{array}{l}\text { Por cálculo f(cultivo, suelo y demanda } \\
\text { atmosférica) }\end{array}$ & (22) \\
\hline Curva retención hídrica & $\theta(\psi)$ & $\begin{array}{l}\text { Cámara de presión Richards } \\
15 \text { cilindros: } 5 \text { tensiones } \times 3 \text { cilindros }\end{array}$ & (14) \\
\hline $\begin{array}{l}\text { Resistencia mecánica a } \\
\text { la penetración } \\
\text { Valor crítico: } 3,6 \mathrm{MPa}\end{array}$ & $\theta \mathrm{RP}(\theta)$ & $\begin{array}{l}\text { Penetrómetro punta cónica } 4 \mathrm{~mm} \\
\text { diámetro y } 30^{\circ} \text { semiángulo } \\
15 \text { cilindros: } 5 \text { tensiones } \times 3 \text { cilindros }\end{array}$ & (22) \\
\hline Intervalo hídrico óptimo & $\mathrm{IHO}$ & $\begin{array}{l}\text { Por cálculo } \\
\mathrm{IHO}=\text { Mín }\left(\theta_{\mathrm{Cc}} ; \theta_{\mathrm{a}}\right)-\text { Máx }\left(\theta_{\mathrm{RP}} ; \theta_{\mathrm{FU}}\right)\end{array}$ & (22) \\
\hline Densidad crítica & Ds crít & Densidad a la que el $\mathrm{IHO}=0$ & (27) \\
\hline Conductividad hidráulica & $\mathrm{K}_{\mathrm{s}}, \mathrm{K}_{0,3}$ & $\begin{array}{l}\text { Infiltrómetro de tensión a } 0 \text { y } 0,3 \text { kPa. } \\
6 \text { repeticiones }\end{array}$ & $(1,7)$ \\
\hline
\end{tabular}

El IHO (22) es el contenido hídrico del suelo dentro del cual: 1) el agua es fácilmente utilizable por el cultivo (Agua retenida a $0,17 \mathrm{MPa}), 2$ ) la masa sólida del suelo es horadable por las raíces (3,6 MPa) y 3) la aireación no es limitante para la respiración radical (15\% de porosidad de aireación). Integra así tres variables: Porosidad de aireación $\left(\theta_{\mathrm{a}}\right)$, Agua fácilmente utilizable $\left(\theta_{\mathrm{FU}}\right)$ y Resistencia mecánica a la penetración radical $\left(\theta_{\mathrm{RP}}\right)$, de modo que en dicho intervalo las raíces no tienen mayores dificultades para ocupar volúmenes crecientes de suelo, ni para absorber agua y disponen de oxígeno suficiente.

El IHO no refiere a la capacidad de la estructura edáfica para conducir fluidos (conductividad hidráulica) y para perdurar (estabilidad); por esto además se han medido las otras propiedades.
El uso de tensioinfiltrómetros permite evaluar la conductividad hidráulica saturada (Ks) y la conductividad por poros menores a $1 \mathrm{~mm}(\mathrm{~K} 3)$. Para determinar el porcentaje de agua que es conducida por distintos tamaños de poros se calcula: por poros $>$ $1000 \mu \mathrm{m}=(\mathrm{Ks}-\mathrm{K} 3) / \mathrm{Ks} \times 100$ y por poros $<$ $1000 \mu \mathrm{m}=\mathrm{K} 3 / \mathrm{Ks} \times 100$ según (8).

La estabilidad de los agregados se determinó con la técnica de Monnier et al. (16).

Eol y Ea hacen referencia a la estabilidad de los agregados al agua después de pretratamiento en alcohol o sólo en agua. El porcentaje de agregados que se rompe por dilatación diferencial, hinchamiento y dispersión es 100- Eol. La contribución del estallido sólo es Eol-Ea (18).

Cuando se agrega PS, se adicionan sales (floculante) y sodio (dispersante). Para estimar si el "ambiente edáfico" es más esta- 
ble por las sales o más disperso se usan los límites propuestos por Rhoades (23), quien plantea una relación entre la CEes y el PSI (Porcentaje de Sodio Intercambiable) a partir del cual el sodio tendría efecto físico negativo, reduciendo la conductividad hidráulica. En el trabajo se ajustó la siguiente ecuación para reconocer el PSI crítico, es decir a partir del cual domina la dispersión sobre la floculación (PSIcrit $=10,7 \times$ CEes $-2,1)$. Por otro lado hay pasaje de maquinaria con suelo mojado, especialmente al aplicar altas dosis de PS. Esto puede conducir al amasado: menos macroporos y más microporos pequeños; además de pérdida de conexión entre poros.

\section{Análisis de los datos}

En todos los casos se evaluó la normalidad de la distribución usando los test: chi-cuadrado de bondad de ajuste, $\mathrm{W}$ de Shapiro-Wilks y Z para curtosis y asimetría. Se usó logaritmo natural para normalizar la conductividad hidráulica y $[\pi / 2 \operatorname{arcsen}(\mathrm{x})$ $\wedge 0,5]$ para el IHO. Posteriormente se identificaron datos atípicos o extraños usando el test de Grubbs y el de Dixon: ambos asumen normalidad. Cuando hubo datos atípicos se los reemplazó por el valor medio obtenido sin incluir el atípico. Por último se realizó el análisis de varianza y comparación de medias con el test de Tuckey ( $\alpha=5 \%$ ).

\section{RESULTADOS Y DISCUSIÓN}

La composición del PS varió notablemente entre el utilizado antes del maíz y de la alfalfa, esto concuerda con lo informado en (5). En el Tabla 5 se puede ver el aporte de minerales para cada dosis utilizada y las diferencias en composición aún para una misma dosis, en la secuencia maíz-alfalfa. Esto destaca la necesidad de analizar la composición química del PS antes de utilizarlo. Nótese además que, con respecto a la aplicación de $100 \mathrm{~kg}$ de FDA, el aporte de $\mathrm{N}$ se logra con dosis medias a bajas, en cambio el aporte de P se alcanza con la menor dosis.

Los cambios en la composición química entre los PS utilizados son grandes en $\mathrm{N}$ y $\mathrm{S}$; intermedios en $\mathrm{Ca}$ y $\mathrm{Na}$ y menores en $\mathrm{P}$, $\mathrm{K}$ y Cl.

El PS aplicado es ácido con pH entre 4,5 a 5; con bajo contenido de sólidos totales: 11 y $13 \%$ y elevada salinidad: CE de $14 \mathrm{dS} / \mathrm{m}$.

Tabla 5: Aportes de $\mathrm{N}, \mathrm{P}, \mathrm{K}, \mathrm{S}, \mathrm{Ca}, \mathrm{Mg}, \mathrm{Na}$ y Cl del abono comercial fosfato diamónico (FDA) y del permeado de suero (PS) por dosis aplicada en dos momentos antes del maíz y de alfalfa; centro este de Córdoba (Argentina) $(30,60,90$ y 120 son $\mathrm{m} 3 / \mathrm{ha})$.

\begin{tabular}{|l|c|c|c|c|c|c|c|c|}
\hline $\begin{array}{l}\text { Compuesto } \\
\text { Tratamiento }\end{array}$ & $\begin{array}{c}\mathrm{N} \\
(\mathrm{kg} / \mathrm{ha})\end{array}$ & $\begin{array}{c}\mathrm{P} \\
(\mathrm{kg} / \mathrm{ha})\end{array}$ & $\begin{array}{c}\mathrm{K} \\
(\mathrm{kg} / \mathrm{ha})\end{array}$ & $\begin{array}{c}\mathrm{S} \\
(\mathrm{kg} / \mathrm{ha})\end{array}$ & $\begin{array}{c}\mathrm{Ca} \\
(\mathrm{kg} / \mathrm{ha})\end{array}$ & $\begin{array}{c}\mathrm{Mg} \\
(\mathrm{kg} / \mathrm{ha})\end{array}$ & $\begin{array}{c}\mathrm{Na} \\
(\mathrm{Na} \mathrm{kg} / \mathrm{ha})\end{array}$ & $\begin{array}{c}\mathrm{Cl} \\
(\mathrm{Cl} \mathrm{kg} / \mathrm{ha})\end{array}$ \\
\hline PS30 maíz & 6 & 27 & 112 & 26 & 29 & 6 & 17 & 64 \\
PS30 alfalfa & 22 & 38 & 89 & 4 & 54 & 6 & 11 & 76 \\
\hline PS60 maíz & 12 & 54 & 223 & 51 & 58 & 12 & 88 & 127 \\
PS60 alfalfa & 44 & 75 & 178 & 8 & 108 & 12 & 23 & 153 \\
\hline PS120 maíz & 24 & 108 & 446 & 102 & 116 & 24 & 69 & 255 \\
\hline PS 90 alfalfa & 88 & 150 & 356 & 16 & 216 & 24 & 34 & 229 \\
\hline FDA maíz & 18 & 20 & - & - & - & - & - & - \\
\hline
\end{tabular}


El resultado de los análisis químicos de suelo se presenta en el Tabla 6. Antes de iniciarse el ensayo el suelo tiene un nivel bajo de $\mathrm{MO}$ y $\mathrm{Nt}$, intermedio a bajo de $\mathrm{P}$ y medio a alto de $\mathrm{Ca}, \mathrm{Mg}$ y $\mathrm{K}$. Se observa que aumenta en profundidad el $\mathrm{Na}, \mathrm{pH}$ y CEes siendo restrictivo para algunos cultivos (19). La MO y el Nt disminuyen en profundidad.

Si bien PSI es elevado debajo de los 15 $\mathrm{cm}$, no supera el límite crítico para la dispersión ya que también hay un alto nivel salino (floculante).

\section{Respuesta de los cultivos}

Maíz: El rendimiento en granos de maíz fue de medio a bajo ya que se cosechó an- tes de madurez fisiológica (maíz para silo), pero la producción de biomasa aérea fue elevada, evidenciando un ciclo de crecimiento favorable y que el suelo no ofreció mayores limitaciones. No hay diferencias significativas entre $\mathrm{T}$ y FDA, aunque este último rindió más (Tabla 7).

La aplicación de PS hizo decrecer el rendimiento. Este descenso podría deberse a un efecto combinado de: 1) el incremento de Na debido al PS que podría ser tóxico ya que este cultivo es sensible (19), 2) un desbalance entre la lactosa y el $\mathrm{N}$ del PS aplicado al suelo, pudiendo causar deficiencia en el crecimiento de los cultivos. La relación $\mathrm{C} / \mathrm{N}$ es muy alta, más de 200 con una fuente de C muy lábil, lo que pue-

Tabla 6: Análisis químico del Natrustol típico, Arroyo Algodón, a diferentes profundidades pre siembra de maíz para silo (campaña 2006-2007) antes de la aplicación de permeado de suero. Valores entre paréntesis corresponden al porcentaje de sodio intercambiable crítico (por encima de él predomina dispersión).

\begin{tabular}{|c|c|c|c|}
\hline Determinación & $\begin{array}{c}0-5 \\
(\mathrm{~cm})\end{array}$ & $\begin{array}{l}5-15 \\
(\mathrm{~cm})\end{array}$ & $\begin{array}{c}15-30 \\
(\mathrm{~cm})\end{array}$ \\
\hline Materia orgánica (\%) & 2,7 & 1,8 & 1,3 \\
\hline Nitrógeno total (\%) & 0,16 & 0,10 & 0,07 \\
\hline Fósforo extraíble (ppm) & 18 & 10 & 10 \\
\hline Calcio intercambiable (cmol/kg) & 9,3 & 7,3 & 10,2 \\
\hline$\%$ de $\mathrm{CIC}$ & 60 & 61 & 52 \\
\hline \multirow[t]{2}{*}{ Magnesio intercambiable (cmol/kg) } & 1,9 & 1,9 & 2,1 \\
\hline & 12 & 16 & 11 \\
\hline \multirow[t]{2}{*}{ Sodio intercambiable $(\mathrm{cmol} / \mathrm{kg})$} & 0,6 & 1,0 & 3,6 \\
\hline & $4(14)$ & $8(18)$ & $18(42)$ \\
\hline \multirow[t]{2}{*}{ Potasio intercambiable (cmol/kg) } & 2,5 & 2,0 & 2,4 \\
\hline & 16 & 17 & 12 \\
\hline Capacidad intercambio Catiónico (cmol/kg) & 15,4 & 12 & 19,5 \\
\hline Conductividad eléctrica extracto de saturación (dS/m) & 1,5 & 1,9 & 4,1 \\
\hline $\mathrm{pH}$ & 6,6 & 7,3 & 8,9 \\
\hline
\end{tabular}


de inmovilizar el $\mathrm{N}$ disponible para el cultivo. Tampoco se encontraron incrementos significativos en (3) en rendimiento ni en biomasa aérea de maíz para silo habiendo aplicado dosis de 17; 52 y $104 \mathrm{~m}^{3} \mathrm{PS} / \mathrm{ha}$, en un Argiudol del centro de Santa Fe. El rendimiento y la biomasa aérea fueron similares a los de este ensayo.
Alfalfa: La producción de alfalfa al cabo de 7 cortes (17 meses post siembra) osciló entre 8000 y $9000 \mathrm{~kg} \mathrm{MS} / \mathrm{ha}$ (Tabla 8). No hubo diferencias significativas entre tratamientos.

Tabla 7: Producción de biomasa aérea y rendimiento en grano de maiz para silo. Centro este de Córdoba (Argentina) en suelo Natrustol típico: ensayo respuesta a la aplicación de permeado de suero (PS). Tratamientos: T, PS30, PS60, PS120 y FDA (100 kg/ha de fosfato diamónico sin PS); T es testigo sin aplicación de PS; 30, 60 y 120 son $\mathrm{m}^{3}$ PS /ha. (Letras distintas indican diferencias significativas al nivel del 5\%)

\begin{tabular}{|c|c|c|}
\hline Tratamientos & $\begin{array}{c}\text { Rendimiento en granos } \\
\text { (kg MS/ha) }\end{array}$ & $\begin{array}{c}\text { Producción de biomasa } \\
\text { aérea (kg MS/ha) }\end{array}$ \\
\hline T & $5.915 \mathrm{a}$ & $23.275 \mathrm{a}$ \\
\hline PS30 & $5.512 \mathrm{ab}$ & $20.642 \mathrm{a}$ \\
\hline PS60 & $5.377 \mathrm{~b}$ & $20.018 \mathrm{~b}$ \\
\hline PS120 & $4.855 \mathrm{~b}$ & $19.859 \mathrm{~b}$ \\
\hline FDA & $6.728 \mathrm{ac}$ & $24.310 \mathrm{a}$ \\
\hline
\end{tabular}

Tabla 8: Producción de alfalfa para forraje, acumulada en 7 cortes (17 meses post siembra). Centro este de Córdoba (Argentina) en suelo Natrustol típico: ensayo respuesta a la aplicación de permeado de suero (PS). Tratamientos: T, PS30, PS60, PS90 y FDA (100 kg/ha de fosfato

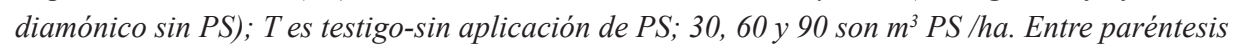
desvio estándar. (Letras distintas indican diferencias significativas al nivel del 5\%)

\begin{tabular}{|c|c|}
\hline Tratamientos & $\begin{array}{c}\text { Producción de alfalfa } \\
(\mathrm{kg} \mathrm{MS} / \mathrm{ha})\end{array}$ \\
\hline $\mathrm{T}$ & $7.937 \mathrm{a}( \pm 1314)$ \\
\hline PS30 & $8.221 \mathrm{a}( \pm 966)$ \\
\hline PS60 & $8.775 \mathrm{a}( \pm 1104)$ \\
\hline PS90 & $8.999 \mathrm{a}( \pm 697)$ \\
\hline FDA & $8.693 \mathrm{a}( \pm 1195)$ \\
\hline
\end{tabular}




\section{Cambios en el suelo}

Propiedades químicas post aplicación de PS al maíz: Al comparar el $\mathrm{T}$ con la menor y mayor dosis de PS aplicado, los resultados del análisis químico del suelo después de cosechado el maíz (Tabla 9) muestran que:

a) No hay diferencias significativas en $\mathrm{MO}, \mathrm{Nt}, \mathrm{Ki}, \mathrm{CIC}$, CEes;

b) El P aumenta de 0 a $5 \mathrm{~cm}$ en PS30 y hasta los $30 \mathrm{~cm}$ en PS120. Si se expresa en $\mathrm{kg}$ $\mathrm{P} /$ ha hasta esa profundidad, incrementa en 46 $\mathrm{kg} /$ ha respecto del $\mathrm{T}$, cifra inferior a los 108 $\mathrm{kg} /$ ha adicionados (Tabla 6) lo que indica que parte del $\mathrm{P}$ aplicado no lo extrae el cultivo y que la técnica analítica de Bray y Kurtz no lo valora totalmente. Nótese que, si bien se aplicó en superficie también aumenta en profundidad, lo que evidencia movilidad de parte del P aplicado: esto importa porque si se aplica en forma no controlada podría contaminar fuentes de aguas subterráneas o superficiales;

c) El Cai aumenta de 0 a $5 \mathrm{~cm}$ en PS120; representando $130 \mathrm{~kg} \mathrm{Ca} /$ ha más que en el T, valor muy próximo a lo aplicado (Tabla 5);

d) El Nai aumenta significativamente en los primeros $15 \mathrm{~cm}$ en PS120, lo que equivale a $207 \mathrm{~kg} \mathrm{Na} / \mathrm{ha}$, valor muy superior a lo adicionado $-69 \mathrm{~kg} \mathrm{Na} / \mathrm{ha}$ (Tabla 5). No se encontró explicación a esta notable diferencia;

e) El Mgi disminuye hasta los 15 cm en PS120, desplazado por $\mathrm{Na}$ y $\mathrm{Ca}$;

f) La reacción del suelo aumenta de 0 a 15 cm cuando se aplica PS pero sin alcalinizarse.

Propiedades químicas post aplicación de PS a la alfalfa y maíz: Los resultados después de la dosis de PS aplicada antes del maíz y repitiendo antes de la alfalfa, al año de implantado este cultivo (Tabla 10) muestran que:

a) No hay diferencias significativas en $\mathrm{MO}, \mathrm{Ki}, \mathrm{CIC}, \mathrm{CEes}$; b) El Nt aumenta hasta los $30 \mathrm{~cm}$ en PS90, la diferencia con el T equivale a 1.820 $\mathrm{kg} \mathrm{N} / \mathrm{ha}$ valor muy superior a lo aplicado (PS120+PS90 -Tabla 5) que sólo fue de 132 $\mathrm{kg} \mathrm{N} / \mathrm{ha}$. ¿Qué ha ocurrido? Es una extraña evidencia que, por su importancia, debería orientar a futuras indagaciones. También se observa que las formas de $\mathrm{N}$ adicionadas pueden lixiviarse ya que se aplica en superficie pero se detectan importantes aumentos hasta los $30 \mathrm{~cm}$, alertando sobre un posible riesgo de contaminación si no se controla;

c) El P también aumenta de 0 a $5 \mathrm{~cm}$ en ambas aplicaciones, llegando hasta los 15 $\mathrm{cm}$ en la dosis más alta, lo que equivale a poco menos de $40 \mathrm{~kg} \mathrm{P} / \mathrm{ha}$. Si se compara con los $258 \mathrm{~kg} \mathrm{P} / \mathrm{ha}$ aplicado en ambas oportunidades (Tabla 6), lo detectado en el análisis del suelo es mucho menos -incluso considerando lo que puede haberse extraído con los cultivos: hay formas de P que no son extraídas por la técnica Bray y Kurtz. También, al igual que el $\mathrm{N}$, se registran incrementos en profundidad denotando movilidad en el perfil y, por tanto, riesgo de contaminación ante aplicaciones descontroladas;

d) El Cai aumenta significativamente sólo en superficie;

e) El Mgi disminuye significativamente;

f) El Nai aumenta significativamente hasta $15 \mathrm{~cm}$ en PS90;

g) $\mathrm{El} \mathrm{pH}$ disminuye de 0 a $5 \mathrm{~cm}$ de profundidad, en ambos, mostrando un comportamiento errático ya que en el cultivo anterior había aumentado.

En (3) aplicaron dosis de 17, 52 y 104 $\mathrm{m}^{3} \mathrm{PS} /$ ha a un maíz para silo sobre un Argiudol del centro de Santa Fe midiendo un incremento del Nt (entre 200 y $450 \mathrm{~kg} \mathrm{~N} /$ ha para PS104), P (15 a $20 \mathrm{~kg} \mathrm{P} / \mathrm{ha}), \mathrm{y} \mathrm{Na}$ en superficie (50 a $200 \mathrm{~kg} \mathrm{Na} / \mathrm{ha}$ ): tendencia similar a la encontrada en este trabajo. 
Tabla 9: Resultados análisis químico de suelos Establecimiento "La Porteña" Arroyo Algodón-lote 26- a la cosecha de maíz para silo en el centro este de Córdoba (Argentina) sobre suelo Natrustol típico: ensayo respuesta a la aplicación de permeado de suero (PS). Tratamientos: T, PS30 y PS120. $T$ es testigo -sin aplicación de PS, 30 y 120 son $\mathrm{m}^{3}$ de PS /ha. (Se comparan sólo pares de valores: letras minúsculas T vs PS30; Tvs. PS120; letra mayúscula PS30 vs PS120; letras distintas indican diferencias significativas al nivel del 5\%; $n=30$ submuestras). Valores entre paréntesis en Na corresponden al porcentaje de sodio intercambiable crítico, a partir del cual comienza la dispersión.

\begin{tabular}{|c|c|c|c|c|c|c|c|}
\hline \multicolumn{2}{|l|}{ Determinaciones } & $\mathrm{T}$ & PS30 & PS120 & $\begin{array}{c} \\
(\%)\end{array}$ & $\begin{array}{l}\text { PS30 } \\
(\%)\end{array}$ & $\begin{array}{l}\text { PS120 } \\
(\%)\end{array}$ \\
\hline \multirow{3}{*}{$\begin{array}{l}\text { Materia orgánica } \\
(\%)\end{array}$} & $0-5$ & $2,9 a$ & $2,5 \mathrm{aA}$ & $2,8 \mathrm{aA}$ & & & \\
\hline & $5-15$ & $2,0 \mathrm{a}$ & $2,0 \mathrm{aA}$ & $2,3 \mathrm{aA}$ & & & \\
\hline & $15-30$ & $1,2 \mathrm{a}$ & $1,3 \mathrm{aA}$ & $1,4 \mathrm{aA}$ & & & \\
\hline \multirow{3}{*}{$\begin{array}{l}\text { Nitrógeno total } \\
(\%)\end{array}$} & $0-5$ & $0,14 a$ & $0,13 \mathrm{aA}$ & $0,14 \mathrm{aA}$ & & & \\
\hline & $5-15$ & $0,09 a$ & $0,09 \mathrm{aA}$ & $0,10 \mathrm{aA}$ & & & \\
\hline & $15-30$ & $0,09 a$ & $0,1 \mathrm{aA}$ & $0,09 \mathrm{aA}$ & & & \\
\hline \multirow{3}{*}{$\begin{array}{l}\text { Fósforo extraíble } \\
\text { (ppm) }\end{array}$} & $0-5$ & $18 \mathrm{a}$ & $35 \mathrm{bA}$ & $67 \mathrm{bB}$ & & & \\
\hline & $5-15$ & $10 \mathrm{a}$ & $10 \mathrm{aA}$ & $15 \mathrm{bB}$ & & & \\
\hline & $15-30$ & $8 \mathrm{a}$ & $8 \mathrm{aA}$ & $12 \mathrm{bB}$ & & & \\
\hline \multirow{3}{*}{$\begin{array}{l}\text { Calcio intercambiable } \\
(\mathrm{cmol} / \mathrm{kg})\end{array}$} & $0-5$ & $9,1 \mathrm{a}$ & $9,3 \mathrm{aA}$ & $10,1 \mathrm{bB}$ & 60 & 62 & 65 \\
\hline & $5-15$ & $7,0 \mathrm{a}$ & $6,8 \mathrm{aA}$ & $7,1 \mathrm{aA}$ & 56 & 54 & 55 \\
\hline & $15-30$ & $9,1 \mathrm{a}$ & $8,7 \mathrm{aA}$ & $9,0 \mathrm{aA}$ & 48 & 46 & 49 \\
\hline \multirow{3}{*}{$\begin{array}{l}\text { Magnesio intercambiable } \\
(\mathrm{cmol} / \mathrm{kg})\end{array}$} & $0-5$ & $1,6 \mathrm{a}$ & $1,6 \mathrm{aA}$ & $1,2 \mathrm{bB}$ & 11 & 11 & 8 \\
\hline & $5-15$ & $1,8 \mathrm{a}$ & $1,5 \mathrm{aA}$ & $1,1 \mathrm{bA}$ & 14 & 12 & 9 \\
\hline & $15-30$ & $2,4 \mathrm{a}$ & $3,0 \mathrm{aA}$ & $2,9 \mathrm{aA}$ & 13 & 16 & 16 \\
\hline \multirow[t]{3}{*}{$\begin{array}{l}\text { Sodio intercambiable } \\
(\mathrm{cmol} / \mathrm{kg})\end{array}$} & $0-5$ & $0,5 \mathrm{a}$ & $0,5 \mathrm{aA}$ & $0,9 \mathrm{bB}$ & $\begin{array}{c}3 \\
(8)\end{array}$ & $\begin{array}{c}3 \\
(6)\end{array}$ & $\begin{array}{c}6 \\
(12)\end{array}$ \\
\hline & $5-15$ & $1,4 \mathrm{a}$ & $1,3 \mathrm{aA}$ & $2,1 \mathrm{bB}$ & 11 & 10 & 16 \\
\hline & $15-30$ & $3,8 \mathrm{a}$ & $4,5 \mathrm{aA}$ & $4,0 \mathrm{aA}$ & 20 & 24 & 22 \\
\hline \multirow[t]{3}{*}{$\begin{array}{l}\text { Potasio intercambiable } \\
(\mathrm{cmol} / \mathrm{kg})\end{array}$} & $0-5$ & $2,4 a$ & $2,6 \mathrm{aA}$ & $2,6 \mathrm{aA}$ & 16 & 17 & 17 \\
\hline & $5-15$ & $2,1 \mathrm{a}$ & $1,9 \mathrm{aA}$ & $2,0 \mathrm{aA}$ & 17 & 15 & 16 \\
\hline & $15-30$ & $2,8 \mathrm{a}$ & $2,7 \mathrm{aA}$ & $2,6 \mathrm{aA}$ & 15 & 14 & 14 \\
\hline \multirow{3}{*}{$\begin{array}{l}\text { Capac. interc. catiónico } \\
(\mathrm{cmol} / \mathrm{kg})\end{array}$} & $0-5$ & $15,1 a$ & $15,0 \mathrm{aA}$ & 15,6 aA & & & \\
\hline & $5-15$ & $12,6 \mathrm{a}$ & $12,5 \mathrm{aA}$ & 12,9 aA & & & \\
\hline & $15-30$ & $19,1 \mathrm{a}$ & $19,1 \mathrm{aA}$ & 18,3 aA & & & \\
\hline \multirow{3}{*}{$\begin{array}{l}\text { Salinidad } \\
\text { Conduct. Eléctrica. Extr. } \\
\text { saturación (dS/m) }\end{array}$} & $0-5$ & $0,9 a$ & $0,8 \mathrm{aA}$ & $1,3 \mathrm{aA}$ & & & \\
\hline & $5-15$ & $0,9 a$ & $0,9 \mathrm{aA}$ & $0,8 \mathrm{aA}$ & & & \\
\hline & $15-30$ & $3,5 \mathrm{a}$ & $4,3 \mathrm{aA}$ & $3,4 \mathrm{aA}$ & & & \\
\hline \multirow[t]{3}{*}{$\mathrm{pH}$} & $0-5$ & $6,6 \mathrm{a}$ & $7,1 \mathrm{bA}$ & $7,0 \mathrm{bA}$ & & & \\
\hline & $5-15$ & $7,3 \mathrm{a}$ & $7,7 \mathrm{bA}$ & $7,7 \mathrm{bA}$ & & & \\
\hline & $15-30$ & $8,7 \mathrm{a}$ & $8,8 \mathrm{aA}$ & $8,6 \mathrm{aA}$ & & & \\
\hline
\end{tabular}


Respuesta de cultivos y suelo al permeado de suero

Tabla 10: Resultados de análisis químico de suelo al año de implantada la alfalfa en el centro este de Córdoba sobre suelo Natrustol: ensayo respuesta a la aplicación de permeado de suero (PS) Tratamientos: T, P30 y P90. T es testigo -sin aplicación de PS, $30 y 90$ son $\mathrm{m} 3$ de PS /ha. (Se comparan sólo pares de valores: letras minúsculas T vs PS30; T vs. PS90; letra mayúscula PS30 vs PS90; letras distintas indican diferencias significativas al nivel del 5\%). Valores entre paréntesis en Nai corresponden al porcentaje de sodio intercambiable crítico, a partir del cual comienza la dispersión.

\begin{tabular}{|c|c|c|c|c|c|c|c|}
\hline \multicolumn{2}{|l|}{ Determinaciones } & $\mathbf{T}$ & PS30 & PS90 & $\mathbf{T}$ & PS30 & PS90 \\
\hline \multirow{3}{*}{$\begin{array}{l}\text { Materia orgánica } \\
(\%)\end{array}$} & $0-5$ & $2,8 \mathrm{a}$ & $2,5 \mathrm{aA}$ & $3,0 \mathrm{aA}$ & & & \\
\hline & $5-15$ & $2,0 \mathrm{a}$ & $1,7 \mathrm{aA}$ & $2,1 \mathrm{aA}$ & & & \\
\hline & $15-30$ & $1,5 \mathrm{a}$ & $1,5 \mathrm{aA}$ & $1,7 \mathrm{aA}$ & & & \\
\hline \multirow{3}{*}{$\begin{array}{l}\text { Nitrógeno total } \\
\text { (\%) }\end{array}$} & $0-5$ & $0,16 a$ & $0,18 \mathrm{aA}$ & $0,21 \mathrm{bB}$ & & & \\
\hline & 5-15 & $0,10 \mathrm{a}$ & 0,09 aA & $0,14 \mathrm{bB}$ & & & \\
\hline & $15-30$ & $0,08 \mathrm{a}$ & 0,08 aA & $0,13 \mathrm{bB}$ & & & \\
\hline \multirow{3}{*}{$\begin{array}{l}\text { Fósforo extraíble } \\
\text { (ppm) }\end{array}$} & $0-5$ & $16 \mathrm{a}$ & $50 \mathrm{bA}$ & $61 \mathrm{bA}$ & & & \\
\hline & $5-15$ & $12 \mathrm{a}$ & $14 \mathrm{aA}$ & $17 \mathrm{bA}$ & & & \\
\hline & $15-30$ & $10 \mathrm{a}$ & $10 \mathrm{aA}$ & $12 \mathrm{aA}$ & & & \\
\hline \multirow{3}{*}{$\begin{array}{l}\text { Calcio intercambiable } \\
(\mathrm{cmol} / \mathrm{kg})\end{array}$} & $0-5$ & $9,2 \mathrm{a}$ & $9,1 \mathrm{aA}$ & $10,5 \mathrm{bB}$ & 62 & 67 & 71 \\
\hline & 5-15 & $7,4 \mathrm{a}$ & $7,1 \mathrm{aA}$ & $8,0 \mathrm{aA}$ & 61 & 61 & 63 \\
\hline & $15-30$ & $10,0 \mathrm{a}$ & 10,3 aA & 10,5 aA & 52 & 55 & 55 \\
\hline \multirow{3}{*}{$\begin{array}{l}\text { Magnesio intercambiable } \\
(\mathrm{cmol} / \mathrm{kg})\end{array}$} & $0-5$ & $1,7 \mathrm{a}$ & $1,8 \mathrm{aA}$ & 1,6 aA & 11 & 13 & 11 \\
\hline & 5-15 & $1,9 \mathrm{a}$ & $1,6 \mathrm{aA}$ & $1,3 \mathrm{bB}$ & 16 & 14 & 10 \\
\hline & $15-30$ & $1,6 \mathrm{a}$ & $1,5 \mathrm{aA}$ & $1 \mathrm{bB}$ & 8 & 8 & 5 \\
\hline \multirow{3}{*}{$\begin{array}{l}\text { Sodio intercambiable } \\
(\mathrm{cmol} / \mathrm{kg})\end{array}$} & $0-5$ & $0,5 \mathrm{a}$ & $0,7 \mathrm{aA}$ & $0,9 \mathrm{bA}$ & $3(3)$ & $4(3)$ & $6(2)$ \\
\hline & 5-15 & $1,4 \mathrm{a}$ & $1,4 \mathrm{aA}$ & $2,1 \mathrm{bB}$ & 12 & 12 & 17 \\
\hline & $15-30$ & $3,8 \mathrm{a}$ & $3,7 \mathrm{aA}$ & $5 \mathrm{aA}$ & 20 & 20 & 26 \\
\hline \multirow{3}{*}{$\begin{array}{l}\text { Potasio intercambiable } \\
(\mathrm{cmol} / \mathrm{kg})\end{array}$} & $0-5$ & $2,5 \mathrm{a}$ & $2,3 a A$ & $2,5 \mathrm{aA}$ & 17 & 17 & 17 \\
\hline & 5-15 & $2,0 \mathrm{a}$ & $1,7 \mathrm{aA}$ & $1,8 \mathrm{aA}$ & 17 & 15 & 14 \\
\hline & $15-30$ & $2,6 \mathrm{a}$ & $2,5 \mathrm{aA}$ & $2,4 \mathrm{aA}$ & 14 & 13 & 13 \\
\hline \multirow{3}{*}{$\begin{array}{l}\text { Capac. interc. catiónico } \\
(\mathrm{cmol} / \mathrm{kg})\end{array}$} & $0-5$ & $14,9 \mathrm{a}$ & $14,1 \mathrm{aA}$ & 14,8 aA & & & \\
\hline & $5-15$ & $12,1 \mathrm{a}$ & $11,7 \mathrm{aA}$ & $12,7 \mathrm{aA}$ & & & \\
\hline & $15-30$ & $19,1 \mathrm{a}$ & $18,7 \mathrm{aA}$ & $19,1 \mathrm{aA}$ & & & \\
\hline \multirow{3}{*}{$\begin{array}{l}\text { Salinidad } \\
\text { Conduct. Eléctrica. Extr. } \\
\text { saturación (dS/m) }\end{array}$} & $0-5$ & $0,5 \mathrm{a}$ & $0,5 \mathrm{aA}$ & $0,4 \mathrm{aA}$ & & & \\
\hline & $5-15$ & $0,4 \mathrm{a}$ & $0,5 \mathrm{aA}$ & $0,6 \mathrm{aA}$ & & & \\
\hline & $15-30$ & $0,5 \mathrm{a}$ & $0,6 \mathrm{aA}$ & $0,4 \mathrm{aA}$ & & & \\
\hline \multirow[t]{3}{*}{$\mathrm{pH}$} & $0-5$ & $6,5 \mathrm{a}$ & $5,9 \mathrm{bA}$ & $6,0 \mathrm{bA}$ & & & \\
\hline & 5-15 & $7,4 \mathrm{a}$ & $7,6 \mathrm{aA}$ & $7,6 \mathrm{aA}$ & & & \\
\hline & $15-30$ & $8,9 \mathrm{a}$ & $8,6 \mathrm{aA}$ & $8,9 \mathrm{aA}$ & & & \\
\hline
\end{tabular}

Revista FAVE - Ciencias Agrarias 18 (1) 2019 


\section{Propiedades físicas post aplicación de PS en maíz}

Estabilidad de agregados (Tabla 11): Después de cosechado el maíz no hubo diferencias en la estabilidad de agregados al agua con pretratamiento en alcohol; sí en cambio se detectó una disminución significativa de la estabilidad de agregados con agua en el tratamiento con mayor aplicación de PS. Nótese la importancia para destruir agregados de la dilatación diferencial, hinchamiento y dispersión: prácticamente la mitad de los agregados se destruyen por estos mecanismos; en cambio el estallido contribuye, adicionalmente, con un $25 \%$ aproximadamente.

En la Tabla 11, si se calcula $\theta \mathrm{s}-\theta 10 \mathrm{kPa}$ se cuantifica la macroporosidad, obteniéndose que PS120 posee un $3 \%$ menos de poros grandes que T, lo que sugiere una modificación estructural que favorece el esta1lido. Además PS120 tiene más Nai (Tabla 9), pero sin sobrepasar el PSI crítico. Las diferencias en estabilidad no pueden atribuirse a un decrecimiento de la MO -factor esencial de cohesión- ya que la misma no ha disminuido (Tabla 9). Nótese que la dispersión, hinchamiento y dilatación por un lado y estallido por el otro no son suficientes para provocar -por sí solos- diferencias significativas. Pero, la diferencia significativa con el T, se expresa cuando se acumulan todos los mecanismos disgregantes.

La dosis menor de PS no presenta diferencias significativas con $\mathrm{T}$ lo que indicaría inocuidad en tal sentido.

Intervalo hídrico óptimo (IHO) (Tabla 12): El T presenta un valor elevado de IHO, según (21) lo que significa condiciones muy buenas para el enraizamiento. Posteriormente, en el cultivo de maíz y hasta su cosecha, por efecto del asentamiento producido por las precipitaciones y el paso de las maquinarias se reduce significativamente; pero aún conserva niveles aceptables. En cambio en PS120 ocurre un deterioro significativo, con un estado físico que puede calificarse sólo como regular. Si se comparan los contenidos hídricos que definen el límite más seco del IHO, $\theta \mathrm{FU}$ o $\theta \mathrm{RP}$; cuando es éste último el que establece el límite, indica que se ha producido una modificación física negativa de deterioro estructural. Esto ocurre en PS120, no así en los otros casos en los que es FU el que establece el límite inferior.

Tabla 11: Estabilidad de agregados del horizonte superficial (0-5cm) de un Natrustol típico. Establecimiento "La Porteña" Arroyo Algodón-lote 26- a la cosecha de maíz para silo en el centro este de Córdoba (Argentina): ensayo respuesta a la aplicación de permeado de suero (PS). Tratamientos: T, PS30 y PS120. Tes testigo -sin aplicación de PS, 30 y 120 son $\mathrm{m} 3$ de PS /ha. (Letras distintas significan diferencias significativas al nivel del 5\% ). DD, H y D es: dilatación diferencial, hinchamiento y dispersión.

\begin{tabular}{|c|c|c|c|c|}
\hline Tratamientos & $\begin{array}{c}\text { Estabilidad } \\
\text { agregados } \\
\text { con alcohol } \\
\%\end{array}$ & $\begin{array}{c}\text { Estabilidad } \\
\text { agregados } \\
\text { con agua } \\
\%\end{array}$ & $\begin{array}{c}\text { Agregados } \\
\text { destruidos por } \\
\text { DD, H y D } \\
\%\end{array}$ & $\begin{array}{c}\text { Agregados } \\
\text { destruidos por } \\
\text { estallido } \\
\%\end{array}$ \\
\hline T & $55 \mathrm{a}$ & $30 \mathrm{a}$ & 45 & 25 \\
\hline P30 & $53 \mathrm{a}$ & $32 \mathrm{a}$ & 47 & 21 \\
\hline P120 & $52 \mathrm{a}$ & $24 \mathrm{~b}$ & 48 & 28 \\
\hline
\end{tabular}


Es importante advertir el deterioro físico evaluado a través de un indicador integral como el IHO, también fue detectado por otro sencillo como la estabilidad de los agregados al agua.

La Figura 1, es útil para utilizar la Ds en el monitoreo de la calidad física de este estrato superficial; se exponen criterios para interpretarla.

1) La Ds se encuentra entre 1,20 y $1,28 \mathrm{~g}$ $\mathrm{cm}^{-3}$; el IHO varía desde 0,17 a 0,12 . La calidad física es "alta" y la degradación pasa de nula a incipiente. El manejo de la condición física del horizonte debería orientarse a mantenerlo dentro de ese intervalo de Ds.

2) Si Ds se encuentra entre 1,28 y 1,36 $\mathrm{g} \mathrm{cm}^{-3}$ la calidad física es media y la degradación moderada. Al encontrar la Ds en este nivel debería servir de advertencia para encontrar las causas que provocan este deterioro y revertirlas; es posible que aún opere la resiliencia natural y sólo baste favorecerla para mejorar la calidad.

3) Valores de Ds mayor a $1,36 \mathrm{~g} \mathrm{~cm}^{-3}$ la calidad es "baja" y la degradación severa: no debería permitirse que los valores de Ds, superen mucho ese valor: la resiliencia edáfica no opera y es difícil revertirla significativamente $\mathrm{y}$ en forma perdurable por medios técnicos.

\section{Propiedades físicas post aplicación de PS a la alfalfa y maíz}

Estabilidad de agregados (Tabla 13):

Después de otra aplicación de PS y transcurridos 17 meses con pastura de alfalfa, se evaluó nuevamente la estabilidad, no se detectaron diferencias excepto en el pretramiento con alcohol donde en la mayor dosis de PS decreció la estabilidad, posiblemente porque aumentó significativamente el Nai (Tabla 10) que supera al PSI crítico.

No debe ignorarse que siempre se presentan indicios de un incipiente daño estructural lo que sugiere utilizar dosis menores que no alteran significativamente a esta importante propiedad.

Conductividad hidráulica (Tabla 14): Se observan los cambios en la conductividad hidráulica del estrato superficial a través del tiempoy conlas aplicaciones máximas dePS.

$\mathrm{T}$ a través del tiempo: Se observa una tendencia a decrecer estadísticamente significativa entre pre siembra del maíz, cuando aún quedaban muchas raíces del cultivo anterior -trigo-, y después de 17 meses de implantada la alfalfa con sucesivos pastoreos. Se destaca la importancia de los poros grandes para conducir el agua ya que entre el 59 y $74 \%$ se mueve a través de ellos.

Tabla 12: Condición física del horizonte superficial (0 a $5 \mathrm{~cm}$ ) de un Natrustol típico. Establecimiento "La Porteña" Arroyo Algodón-lote 26- maíz para silo (campaña 2006-2007): ensayo respuesta a la aplicación de permeado de suero (PS). Tratamientos: T y PS120. T es testigo-sin aplicación de PS; PS120 son $120 \mathrm{~m} 3$ de PS /ha. (letras distintas significan diferencias significativas al nivel del 5\%).

\begin{tabular}{|c|c|c|c|c|c|c|c|c|}
\hline Caso & $\begin{array}{c}\theta \mathrm{S} \\
\left(\mathrm{cm}^{3} \mathrm{~cm}^{-3}\right)\end{array}$ & $\begin{array}{c}\theta \mathrm{a} \\
\left(\mathrm{cm}^{3} \mathrm{~cm}^{-3}\right)\end{array}$ & $\begin{array}{c}\theta 10 \mathrm{kPa} \\
\left(\mathrm{cm}^{3} \mathrm{~cm}^{-3}\right)\end{array}$ & $\begin{array}{c}\theta \mathrm{FU} \\
\left(\mathrm{cm}^{3} \mathrm{~cm}^{-3}\right)\end{array}$ & $\begin{array}{c}\theta \mathrm{RP} \\
\left(\mathrm{cm}^{3} \mathrm{~cm}^{-3}\right)\end{array}$ & $\begin{array}{c}\mathrm{IHO} \\
\left(\mathrm{cm}^{3} \mathrm{~cm}^{-3}\right)\end{array}$ & $\begin{array}{c}\text { Densidad } \\
\text { promedio } \\
\left(\mathrm{g} \mathrm{cm}^{-3}\right)\end{array}$ & $\begin{array}{c}\text { Densidad } \\
\mathrm{crítica}^{\left(\mathrm{g} \mathrm{cm}^{-3}\right)}\end{array}$ \\
\hline $\begin{array}{c}\mathrm{T} \\
\text { pre maíz }\end{array}$ & 0,494 & 0,344 & 0,386 & 0,200 & 0,165 & $0,14 \mathrm{a}$ & 1,29 & 1,45 \\
\hline $\begin{array}{c}\mathrm{T} \\
\text { post maíz }\end{array}$ & 0,490 & 0,340 & 0,414 & 0,224 & 0,225 & $0,11 \mathrm{~b}$ & 1,30 & 1,48 \\
\hline PS120 & 0,486 & 0,336 & 0,410 & 0,236 & 0,257 & $0,08 \mathrm{c}$ & 1,32 & 1,46 \\
\hline
\end{tabular}

Revista FAVE - Ciencias Agrarias 18 (1) 2019 | 


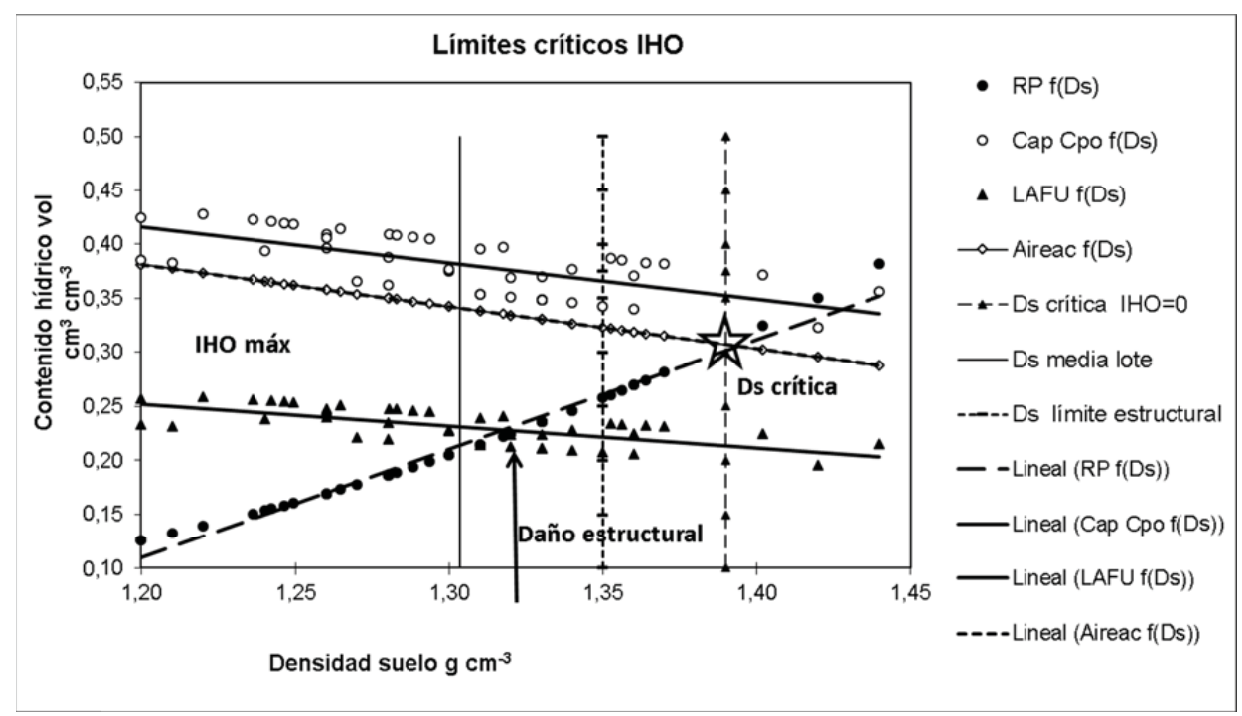

Figura 1: Variación del Intervalo Hídrico Óptimo (IHO) con la densidad del suelo del horizonte superficial (0 a $5 \mathrm{~cm}$ ) de un Natrustol típico, Establecimiento "La Porteña" Arroyo Algodón (Córdoba) -lote 26-: ensayo de respuesta a la aplicación de permeado de suero, se grafica el conjunto de tratamientos. Ds es densidad del suelo; Aireac, Cap Cpo; LAFU, RP, es respectivamente el contenido hídrico volumétrico, cuando hay suficiente Porosidad de Aireación, a Capacidad de Campo, en el Límite de Agua Fácilmente Utilizable y cuando limita la Resistencia a la Penetración. Ds crítica es la densidad a la que el IHO se hace cero.

Tabla 13: Estabilidad de agregados del horizonte superficial (0 a $5 \mathrm{~cm}$ ) de un Natrustol típico. Establecimiento "La Porteña" Arroyo Algodón -lote 26- $2^{\circ}$ año de pradera de alfalfa en el centro este de Córdoba (Argentina): ensayo respuesta a la aplicación de permeado de suero (PS). Tratamientos: T, PS30 y PS90. T es testigo -sin aplicación de PS, 30 y 90 son $\mathrm{m}^{3}$ de PS /ha. (Letras distintas significan diferencias significativas al nivel del $5 \%$ en las columnas). DD, H y D es: dilatación diferencial, hinchamiento y dispersión.

\begin{tabular}{|c|c|c|c|c|}
\hline Tratamientos & $\begin{array}{c}\text { Estabilidad } \\
\text { agregados } \\
\text { con alcohol } \\
\%\end{array}$ & $\begin{array}{c}\text { Estabilidad } \\
\text { agregados } \\
\text { con agua } \\
\%\end{array}$ & $\begin{array}{c}\text { Agregados } \\
\text { destruidos por } \\
\text { DD, H y D } \\
\%\end{array}$ & $\begin{array}{c}\text { Agregados } \\
\text { destruidos por } \\
\text { estallido } \\
\%\end{array}$ \\
\hline T & $80 \mathrm{a}$ & $45 \mathrm{a}$ & 20 & 35 \\
\hline P30 & $77 \mathrm{a}$ & $43 \mathrm{a}$ & 33 & 34 \\
\hline P90 & $72 \mathrm{~b}$ & $48 \mathrm{a}$ & 38 & 24 \\
\hline
\end{tabular}


Tabla 14: Conductividad hidráulica saturada (Ks) e insaturada a tensión 0,3 kPa (K3) y proporción del agua conducida por distintos tamaños de poro en el horizonte superficial de un Natrustol típico, Establecimiento "La Porteña” Arroyo Algodón (Córdoba, Argentina) -lote 26-: ensayo de respuesta a la aplicación de permeado de suero (PS). Tratamientos: T, PS120 y PS120+90. T es testigo -sin aplicación de PS- 120 y 120+90 son $\mathrm{m}^{3}$ de PS /ha. Letras distintas significan diferencias significativas al nivel del 5\%; Comparaciones: con minúscula se comparan los T entre sí; con mayúscula T post maiz vs PS120; con cursiva Ty PS post alfalfa; los guiones indican que no se hizo esa comparación)

\begin{tabular}{|c|c|c|c|c|}
\hline Tratamientos & $\begin{array}{c}\text { Diámetro poro } \\
\text { (micrones) } \\
\text { que conducen agua }\end{array}$ & $\begin{array}{c}\% \\
\text { conducción } \\
\text { agua }\end{array}$ & $\begin{array}{c}\mathrm{Ks} \\
\mathrm{cm} / \mathrm{h}\end{array}$ & $\begin{array}{c}\mathrm{K} 3 \\
\mathrm{~cm} / \mathrm{h}\end{array}$ \\
\hline \multirow{2}{*}{ T Maíz pre siembra } & $>1000$ & 74 & \multirow{2}{*}{$1,9 a--$} & \multirow{2}{*}{$0,5 \mathrm{a}--$} \\
\hline & $<1000$ & 26 & & \\
\hline \multirow{2}{*}{ T Maíz post cosecha } & $>1000$ & 59 & \multirow{2}{*}{$1,7 \mathrm{ab} A-$} & \multirow{2}{*}{0,7 a $\mathrm{A}-$} \\
\hline & $<1000$ & 41 & & \\
\hline \multirow{2}{*}{ T Post 1 año alfalfa } & $>1000$ & 62 & \multirow{2}{*}{$1,3 b-a$} & \multirow{2}{*}{$0,5 a-a$} \\
\hline & $<1000$ & 38 & & \\
\hline \multirow{2}{*}{ PS $_{120}$ Maíz post cosecha } & $>1000$ & 45 & \multirow{2}{*}{$1,1-B-$} & \multirow{2}{*}{$0,6-A-$} \\
\hline & $<1000$ & 55 & & \\
\hline \multirow{2}{*}{$\mathrm{PS}_{120+90}$ Post 1 año alfalfa } & $>1000$ & 38 & \multirow{2}{*}{$0,8--a$} & \multirow{2}{*}{$0,5--a$} \\
\hline & $<1000$ & 62 & & \\
\hline
\end{tabular}

Efecto del permeado post cosecha maíz: 4 meses después de aplicar una dosis elevada se registra un deterioro físico que afecta la trama porosa de mayor tamaño. Por este motivo, en el cultivo siguiente al maíz, en alfalfa, se redujo la dosis a $90 \mathrm{~m}^{3} /$ ha de PS.

Efecto del permeado post alfalfa: 17 meses después de aplicar $90 \mathrm{~m}^{3} /$ ha de PS en el sitio en el que anteriormente se había aplicado $120 \mathrm{~m}^{3} /$ ha se midió un decrecimiento de Ks estadísticamente significativa.

Hay que destacar que esto evalúa la evolución del horizonte superficial partiendo de un estrato con abundantes raíces en descomposición de trigo, pasando por un pe- riodo de asentamiento por precipitaciones y pasaje de maquinarias durante el cultivo de maíz, luego la siembra de alfalfa y midiendo 17 meses después de un largo período sin laboreo y con pisoteo al pastorear.

La conductividad hidráulica por los poros de menor tamaño (K3) en ningún caso se modificó significativamente lo que indica que son los macroporos los que cambian cuando se daña la estructura. 


\section{CONCLUSIONES}

La composición química entre los PS utilizados posee elevado contenido de $\mathrm{N} \mathrm{y}$ $\mathrm{S}$; intermedio en Ca y Na y bajas concentraciones de $\mathrm{P}, \mathrm{K}$ y $\mathrm{Cl}$.

El rendimiento en granos y biomasa aérea de maíz presenta una tendencia lineal decreciente al aumentar las dosis de PS. Se incrementó el $\mathrm{P}$ en todos los tratamientos, aumentando también en profundidad con las dosis mayores, lo que evidencia la movilidad de algunas formas de P. El sodio intercambiable se incrementa en el tratamiento de mayor dosis. El Cai aumenta en superficie. La salinidad y $\mathrm{pH}$ no presentan aumentos de consideración. Sólo el Mgi muestra tendencia a disminuir. La estabilidad de los agregados disminuye con la mayor dosis (P120) al igual que el intervalo hídrico óptimo (IHO) reduciéndose de 0,11 a $0,08 \mathrm{~cm}^{3} / \mathrm{cm}^{3}$; pero esto no ocurre con bajas dosis (P30).

La producción de alfalfa no presentó diferencias significativas con la aplicación de PS. Tampoco hay diferencias importantes en $\mathrm{MO}, \mathrm{Ki}, \mathrm{CIC}$, CEes y $\mathrm{pH}$. En cambio el P, Nt y Nai se incrementan y se distribuyen en profundidad. El Cai aumenta significativamente sólo en superficie y el Mgi disminuye significativamente. Si bien la estabilidad de agregados no muestra diferencias, sí lo hace la Ks.

\section{RECOMENDACIONES}

Evaluar la composición del PS antes de utilizarlo para ajustar las dosis que no deberían pasar los 30 a $60 \mathrm{~m}^{3} / \mathrm{ha}$. Si bien con el agregado de PS no se espera aumento en el rendimiento, se busca la reposición de $\mathrm{P}, \mathrm{N}$ y Ca; sin que haya incrementos indeseables en la salinidad, sodicidad, como tampoco daño físico. Cuando se aplique frecuentemente ( 2 veces por año) dosis de 30 a 60 $\mathrm{m}^{3} / \mathrm{ha}$ de PS, se sugiere evaluar cada 3 años la evolución de las siguientes propiedades: P, Nt, Cai, Mgi, Nai, estabilidad de agregados al agua, Ks y en vez del IHO la Ds. En futuras experiencias, con el objeto de buscar incrementos en el rendimiento de los cultivos se recomienda utilizar lotes con bajo nivel de P (menor de $12 \mathrm{ppm})$ y adicionar abono nitrogenado para prevenir la inmovilización que puede producir la alta relación $\mathrm{C} / \mathrm{N}$ del PS.

\section{BIBLIOGRAFÍA}

1.- ANKENY, M. D.; AHMED, M.; KASPAR, T.C.Y HORTON, R. 1991. Simple field method for determining unsaturated hydraulic conductivity. Soil Sci. Soc. Am. J. 55:467-470.

2.- APHA. 2001. Standard Methods for Examination of Water and Wastewater. 20th Edition. American Public Health Association, Washington DC.

3.- BADINO, O.; PILATTI, M.A.; FELLI, O.; WEIDMANN, P. E.Y GHIBERTO, P. 2011. Permeado de suero como abono: Respuesta de maíz para silo y efectos en un Argiudol de la Pampa llana Santafesina. Revista FAVE. ISSN 1666-7719. Vol. $10 \mathrm{~N}^{\circ}$ 2-77-85.

4.- EPA. United States Environmental Protection Agency. https://www.epa.gov.

Revista FAVE - Ciencias Agrarias 18 (1) 2019 
5.- FELLI, O. M.; BADINO, O.; PI- 13.- JACKSON, M.L. (Ed.). LATTI, M. A. Y ALESSO, C. A. 1982. Análisis químico de sue2012. Caracterización química del los. Omega, Barcelona, 663 pp. permeado de suero como abono. Va- 14.- KLUTE, A. 1986. Water retention: Lariación estacional y por procedencia. Revista FAVE - ISSN 16667719. Ciencias Agrarias 11 (2) 2012.

6.- FORSYTHE, W. 1975. Física de suelos. Manual de laboratorio. IICA. San José de Costa Rica. 212pp.

7.- GHIBERTO, P. J. 1999. Metodologias para a obtençao de parámetros utilizados em modelos de infiltraçao da água no solo. Dissertaçao (Mestrado). ESALQ, USP, Brasil.

8.- GHIBERTO, P. J..; PILATTI, M. A; IMHOFF, S. Y ORELLANA, J. A. DE. 2007. Hydraulic conductivity of Molisolls irrigated with sodic-bicarbonated waters in Santa Fe (Argentina). Agricultural Water Management v.88, n.1-3, p.192 - 200, 2007.

9.- GRASSELLII, M.; NAVARRO, A. A.; FERNÁNDEZ, H. M.; MIRANDA, M. V.; CAMPERI, S.A.Y CASCONE, O. 1997. ¿Qué hacer con el suero del queso? Ciencia Hoy. 8(43): 12-17.

10.- HEBERT, M. 2004. Guide sur la valorisation des materias residuelles fertilizantes- Cráteres de referente et normes reglamentaires. Direction du milieu rural. Environnement Québec.Canadá. Dépôt légal- Bibliotheque nationale du Quebec. ISBN 2-550-42069-1.

11.- IMHOFF, S.; PIRES, A.; SILVA DA Y TORMENA, C.A. 2000. Spatial heterogeneity of soil properties in areas under elephant-grass short-duration grazing system. Plant and Soil 219, 161-168. boratory Methods. In: A. KLUTE (ed). Methods of Soil Analysis - Physical and Mineralogical Methods. America Society of Agronomy. Madison. 635-660.

15.- LOMA, J.L.DE LA. 1980. Experimentación Agrícola. UTEHA, México. 493pp

16.- MONNIER, G. 1965. Action des matières organiques sur la stabilité structurales des sols. Ann. Agron. 16:327-400, 471-574.

17.- MULLA, D.J.Y MCBRATNEY,A.B. 2000. Soil Spatial Varaibility. En Summer M.E: Handbook of S. Sc. A9. 324pp.

18.- PILATTI, M.A. Y ORELLANA, J.A. DE. 2012. Suelos ideales para agricultura sostenible. Revista FAVE sección Ciencias Agrarias, Vol.11 N¹- 65-87.

19.- PILATTI, M.A. Y ORELLANA, J.A. DE. 2016. Hacia una clínica de suelos: Mirando al suelo con ojos de planta. Edic. Colección 'Cátedra' UNL, 280 pp. ISBN: 978-987-657-959-9

20.- PILATTI, M.A.; GHIBERTO, P.J. E IMHOFF, S. 2006. Application of a General Relationship between Soil Particle Density and Organic Matter to Mollisols of Santa Fe (Argentina). $18^{\circ}$ Congreso Mundial de la Ciencia del Suelo. Philadelphia, USA.Trabajo 163-11.

21.- PILATTI, M.A.; ORELLANA, J.A. DE Y FELLI, O. 2003. The Ideal Soil: III) Fitness of Edaphic Variables to Achieve Sustenance in Agroecosystems. J. of Sustainable Agriculture 22 (2): 109-132.

12.- INTA. 1991. Cartas de Suelos de la provincia de Córdoba - Localidad: HOJA JAMES CRAIK. 102 pp. 
22.- PILATTI, M.A.; ORELLANA, J. A. DE; IMHOFF, S. Y SILVA, Á. P. DA. 2012. Actualización de los límites críticos del Intervalo Hídrico Óptimo (IHO). Ciencia del Suelo (Argentina). 30 (1): 9-21.

23.- RHOADES, J. D. 1982. Reclamation and management of salt affects soils after drainage. En Proc. 1er American Western Prov. Conf. Ratios. Water Soil Res. Manag., Canadá, 123-97.

24.- RICHARDS, L. A. (Ed.). 1954. Diagnóstico y rehabilitación de suelos salinos y sódicos. Manual de Agricultura $\mathrm{N}^{\circ}$ 60. Limusa, México.

25.- SAGPYA (Secretaría de Agricultura, Ganadería, Pesca y Alimentación de la Nación Argentina). Dirección de Producción Agrícola. 2004. Sistema de Apoyo Metodológico a los Laboratorios de Análisis de Suelos (SAMLA).

CD-room. ISBN 987-9184-40-8.

26.- SHALLER, A. 2008. Sueros de lechería. Dirección de Industria Alimentaria y Agroindustria. Ministerio de Agricultura, Ganadería y Pesca.

27.- SILVA, A.P. DA; KA, B.D.Y PERFECT, E. 1994. Characterisation of the least limiting water range of soil. Soil Science Society of America Journal 58: 1775-1781. 